\title{
Reconstrucción quirúrgica de dolicoarteriopatía sintomática en la arteria carótida interna y predictores de corrección hemodinámica
}

\section{Surgical reconstruction of symptomatic dolicoarteriopathy in the internal carotid artery and predictors of hemodynamic restoration}

\author{
Erika B. Ramírez-Pérez ${ }^{1 *}$, José J. García-Pérez ${ }^{1}$, Octavio Fernandez-Sandoval' ${ }^{1}$ y \\ Efraín Maldonado-Alcaráz ${ }^{2}$ \\ ${ }^{1}$ Departamento de Angiología y Cirugía Vascular; ${ }^{2}$ Departamento de Urología. Centro Médico Nacional Siglo XXI, Ciudad de México, México
}

\begin{abstract}
Resumen
Objetivo: Determinar los factores predictores que influyen en la reconstrucción quirúrgica de dolicoarteriopatía (DAP) sintomática en la arteria carótida interna ( $A C l)$. Métodos: Análisis retrospectivo de pacientes que fueron sometidos a reconstrucción quirúrgica de DAP tipo kinking o coiling de la ACl durante los meses de enero de 2016 a marzo de 2019. Se revisaron variables demográficas y se realizó un análisis bivariado con la finalidad de determinar la dependencia entre ellas y su influencia en la corrección anatómica, hemodinámica y en la presencia de complicaciones. El procesamiento de datos se realizó con el programa SPSS, se utilizó estadística según correspondiese (prueba de Mann Whitney, $X^{2}, T$ de Student). Se preestableció un valor de $p \leq 0.05$ para significancia estadística. Resultados: Se incluyeron 111 pacientes, la media de edad fue de 73 años, 87 pacientes (78.4\%) pertenecían al sexo femenino. En 68 pacientes (61.2\%), la manifestación inicial fue un trastorno no hemisférico: síncope, 14 casos (12.6\%); tumor hiperpulsátil, 14 casos (12.6\%), y trastornos vestibulares, 13 casos (11.7\%). Los síntomas hemisféricos se presentaron en 43 casos (38.7\%). La DAP más frecuente fue la de tipo 3 , con 97 casos (87.4\%). La coexistencia de enfermedad carotídea se presentó en 47 pacientes (42.3\%). Los factores predictores de la corrección hemodinámica son endarterectomía simultánea $(p=0.016)$ y sangrado transquirúrgico $(p=0.027)$; para la corrección anatómica, la velocidad pico sistólica preoperatoria $130 \mathrm{~cm} / \mathrm{s}$ (rango intercuartil [RIC] 102-155.75) $(p=0.049)$; $y$ para la corrección de ambas sin presencia de complicaciones, índice de masa corporal, obesidad $(p=0.005)$, coexistencia de comorbilidades no cardiovasculares $(p=0.005)$, tipo de sintomatología preoperatoria $(p=0.082)$ y tiempo de pinzamiento carotídeo ( $p=0.045) 30 \mathrm{~min}(R I C$ 23-35). Conclusión: La corrección de las DAP de la ACl es una técnica segura y eficaz. Este estudio demostró que existen factores predictores relacionados con el paciente, la enfermedad y transquirúrgicos que pueden influir en el desenlace postoperatorio.
\end{abstract}

Palabras clave: Dolicoarteriopatías. Endarterectomía. IMC. Tiempo de pinzamiento.

Correspondencia:

*Erika B. Ramírez-Pérez

E-mail: eritrea_33@ hotmail.com
Disponible en internet: 16-04-2020

Rev Mex Angiol. 2020;48(1):1-10 www.RMAngiologia.com

0377-4740/@ 2020 Sociedad Mexicana de Angiología y Cirugía Vascular y Endovascular, A.C. Publicado por Permanyer. Este artículo es de open access bajo la licencia CC BY-NC-ND (http://creativecommons.org/licenses/by-nc-nd/4.0/).

Fecha de recepción: 12-02-2020

Fecha de aceptación: 16-02-2020

DOI: 10.24875/RMA.M20000007 


\section{Abstract}

Objective: To determine the predictive factors that influence the surgical reconstruction of symptomatic dolicoarteriopathies (DAP) in the internal carotid artery (ICA). Methods: Retrospective analysis of patients who underwent surgical reconstruction of (DAP) type kinking or coiling of the ICA from January 2016 to March 2019. Demographic variables were reviewed and a bivariate analysis was performed in order to determine the dependence between them and its influence on anatomical, hemodynamic correction and presence of complications. Data processing was performed with the SPSS program. Statistics were used as appropriate (MannWhitney test, $X^{2}$, Student's T). A value of $p \leq 0.05$ was preset for statistical significance. Results: A total of 111 patients were included, the mean age was 73 years, 87 patients $(78.4 \%)$ were women. In 68 patients $(61.2 \%)$ the initial manifestation was a non-hemispheric disorder: syncope 14 cases (12.6\%), hyperpulsatile tumor 14 (12.6\%) and vestibular disorders 13 cases (11.7\%); hemispheric symptoms occurred in 43 cases (38.7\%), the most frequent DAPs was the type 3 in 97 cases (87.4\%). The coexistence of carotid disease occurred in 47 patients (42.3\%). The predictors of hemodynamic correction are simultaneous endarterectomy $(p=0.016)$ and intraoperative bleeding $(p=0.027)$; anatomical correction: preoperative PSV $130 \mathrm{~cm} / \mathrm{sec}(I Q$ 102-155.75) $(p=0.049)$ and for the correction of both without the presence of complications: BMI, obesity $(p=0.005)$, coexistence of non-cardiovascular comorbidities $(p=0.005)$, type of preoperative symptomatology $(p=0.082)$ and carotid clamp time $(p=0.045) 30 \mathrm{~min}(I Q R$ 23-35). Conclusion: The surgical correction of $D A P$ is a safe and effective technique; this study demonstrates that there are predictive factors related to the patient, the disease and intraoperatively that may influence the postoperative outcome.

Key words: Dolicoarteriopathies. Endarterectomy. BMI. Clamping time.

\section{Introducción}

Las variaciones morfológicas en el curso y la geometría de la arteria carótida interna ( $\mathrm{ACl})$, también conocidas como dolicoarteriopatías (DAP), a menudo, hasta en el $30 \%$ de los casos, se encuentran como hallazgo durante estudios de gabinete por patología de la región cervical' ${ }^{1}$. En general, se considera una enfermedad multifactorial, con una predisposición genética asociada a factores de riesgo cardiovascular que causa engrosamiento de la pared arterial y un trastorno hemodinámico significativo que puede cambiar el flujo sanguíneo dentro del vaso y suponer una causa no infrecuente de evento vascular cerebral ${ }^{2,3}$. Las indicaciones para el tratamiento quirúrgico para la corrección del segmento redundante de la arteria carótida interna no están bien definidas por consensos internacionales, como sucede con la enfermedad carotídea, sin embargo, se ha observado un beneficio claro en los pacientes con: 1) síntomas de isquemia cerebral ipsilateral a la lesión identificada como segmento redundante carotídeo mediante estudio de imagen ${ }^{4}$; 2) coexistencia de estenosis de la arteria carótida interna correspondiente al nivel de la docilidad; 3) ausencia de otras causas de deterioro neurológico tales como enfermedad cardíaca, enfermedades hipertensivas y metabólicas no tratadas, trastornos laberínticos, hipotensión ortostática e incluso espondilosis cervical; 4) ausencia de infarto cerebral irreversible ${ }^{5,6}$; 5) velocidad pico sistólica mayor de $150 \mathrm{~cm} / \mathrm{s}$ y ensanchamiento espectral en el sitio de la lesión; 6) sintomatología hemisférica recurrente aun con uso de tratamiento antiplaquetario, y 7) sintomatología no hemisférica (mareos, visión borrosa, hemianopsia homónima lateral, inestabilidad de la marcha, episodios sincopales transitorios), en caso de presentar dos o más síntomas que afectan la vida diaria y solo después de al menos 3 meses de tratamiento antiplaquetario adecuado 7 . Se tiene conocimiento que la mejor alternativa de tratamiento es la resección del segmento redundante y la reconstrucción vascular, sin embargo, se desconoce cuáles son las variables demográficas asociadas al éxito o fracaso al momento de realizar la corrección quirúrgica. Por lo tanto, el objetivo del estudio fue identificar las variables demográficas y su posible asociación con el resultado perioperatorio en la corrección anatómica, la corrección hemodinámica y la ausencia de complicaciones en una serie consecutiva de pacientes con DAP tipo kinking o coiling de la $\mathrm{ACl}$.

\section{Métodos}

Se realizó un estudio de investigación de tipo observacional, descriptivo, retrospectivo y trasversal en el Hospital Centro Médico Nacional Siglo XXI del Instituto Mexicano del Seguro Social en la Ciudad de México en un periodo de evaluación del 1 de enero de 2016 al 1 de enero de 2019. Esta investigación fue dirigida por el Departamento de Angiología y Cirugía Vascular. Mediante una revisión de expedientes clínicos de pacientes sintomáticos sometidos a reconstrucción 
quirúrgica de DAP en la arteria carótida interna, se caracterizaron las variables demográficas, las cuales se dividieron para su mejor entendimiento en características del paciente, de la enfermedad, posquirúrgicas y las relacionadas con el desenlace postoperatorio. En segundo término, se hizo un análisis bivariado con la finalidad de determinar la dependencia entre variables y su influencia como factor predictor para una corrección anatómica, hemodinámica y la presencia de complicaciones. En cuanto a las características del paciente, se incluyó la edad, el género, índice de masa corporal (IMC), índice tabáquico, comorbilidades de alto riesgo cardiovascular (diabetes mellitus, hipertensión arterial sistémica, cardiopatía isquémica, dislipidemia), otras comorbilidades (patología tiroidea, vasculares, inflamatorias y pulmonares), índice de comorbilidad de Charlson, sintomatología preoperatoria, tiempo del diagnóstico. Respecto a las características de la enfermedad, se incluyó la velocidad pico sistólica (VPS) preoperatoria $(\mathrm{cm} / \mathrm{s})$, lateralidad, tipo de segmento redundante de la $\mathrm{ACl}$, nivel de bifurcación, enfermedad ateromatosa asociada; en cuanto a las características posquirúrgicas, se valoró el tipo de técnica quirúrgica, tiempo de pinzamiento, sangrado transquirúrgico; y para las variables del desenlace postoperatorio, se incluyó la VPS postoperatoria en $\mathrm{cm} / \mathrm{s}$, complicaciones, reestenosis a 36 meses de seguimiento, corrección hemodinámica, corrección anatómica y corrección tanto anatómica y hemodinámica sin presencia de complicaciones. Para el análisis estadístico se realizaron frecuencias, proporciones y $\mathrm{X}^{2}$ para variables categóricas, para variables numéricas se estimó $U$ de Mann-Whitney, excepto para la edad en la que se realizo $T$ de Student, con una $p \leq 0.05$ para la significancia estadística. Se capturó la información en hojas prediseñadas de Excel para su correcto análisis estadístico mediante el programa SPSS versión 25 para Windows.

\section{Resultados}

Una vez realizado el análisis estadístico se obtuvieron los resultados que se muestran a continuación. Se realizó la revisión de 126 expedientes clínicos, con exclusión de 15 casos. Un total de 111 pacientes fueron incluidos en el presente estudio. La media de edad fue de 73 años $( \pm 5)$, la distribución por género se inclinó hacia el sexo femenino, 87 pacientes $(78.4 \%)$. Los factores de riesgo cardiovascular más prevalentes fueron la hipertensión arterial sistémica, presente en 87 casos (78.4\%), el tabaquismo positivo, en 47 casos (42.3\%), y la dislipidemia, en 44 casos (39.6\%). Un índice de comorbilidad de Charlson $40>$ puntos se encontró en 61 casos (55\%). En 78 pacientes (61.2\%), la manifestación inicial fue un trastorno no hemisférico, siendo el síncope en 14 casos (12.6\%), el tumor hiperpulsátil en 14 casos (12.6\%) y los trastornos vestibulares en 13 casos (11.7\%) los tres síntomas principales. Respecto a la sintomatología hemisférica, el ataque isquémico transitorio, con 25 casos (22.5\%), el evento vascular cerebral, con 10 casos (9\%), y la amaurosis fugaz, con 8 casos (7.2\%), fueron los más frecuentes (Fig. 1). El tipo de DAP más frecuente fue el de tipo 3 , con 97 casos $(87.4 \%)$, siendo el grado 1 el más frecuente, presente en 60 casos (54.1\%) (Fig. 2), y coexistiendo la enfermedad ateromatosa en 47 pacientes de los 111 casos (42. 3\%). La bifurcación alta estuvo en 63 casos (56.8\%). El tipo de técnica quirúrgica más frecuentemente realizada fue la anastomosis término-terminal de arteria carótida común a la arteria carótida interna, con 70 casos (63.1\%) (Figs. 3 y 4), siendo la interposición de injerto protésico el tipo de reconstrucción menos frecuentemente, realizado en 2 casos (1.8\%). La mediana de pinzamiento carotídeo fue de $29 \mathrm{~min}$ (RIC 21-35) y el sangrado transquirúrgico fue de $50 \mathrm{ml}$ (RIC 50-100). La VPS postoperatoria fue de $75 \mathrm{~cm} / \mathrm{s}$ (RIC 60-100). Las complicaciones se presentaron en 19 casos (17.1\%), siendo la lesión de nervio (N) laríngeo recurrente en 8 casos (7.2\%). La reestenosis a 36 meses de seguimiento se presentó en 2 casos (1.8\%). La corrección hemodinámica se presentó en 104 casos (93.7\%), la corrección anatómica, en 104 casos (93.7\%), y la corrección de ambas sin presencia de complicaciones, en 85 casos (76.6\%).

Para una corrección hemodinámica se encontraron dos variables estadísticamente significativas, la primera fue la endarterectomía simultánea ( $p=0.016)$, y se encontró que hubo corrección hemodinámica en 75 casos $(97.4 \%)$ en los que no se realizó endarterectomía simultánea, y la segunda fue el sangrado transquirúrgico ( $p=0.027)$, con una mediana de $33 \mathrm{ml}$ (RIC 20-50), de tal forma que a menor sagrado mayor corrección. Para una corrección anatómica, la VPS preoperatoria, con una mediana de $130 \mathrm{~cm} / \mathrm{s}$ (RIC 102-155.75), tuvo una $p=0.049$, y se vio que a mayor repercusión hemodinámica mayor corrección anatómica posquirúrgica. Para una corrección tanto anatómica como hemodinámica sin presencia de complicaciones se encontraron cuatro variables estadísticamente significativas: el IMC ( $p=0.005)$, la obesidad fue un factor predictor, ya que, de los 111 pacientes, 36 casos con obesidad no se curaron (94.7\%); la asociación a otras 


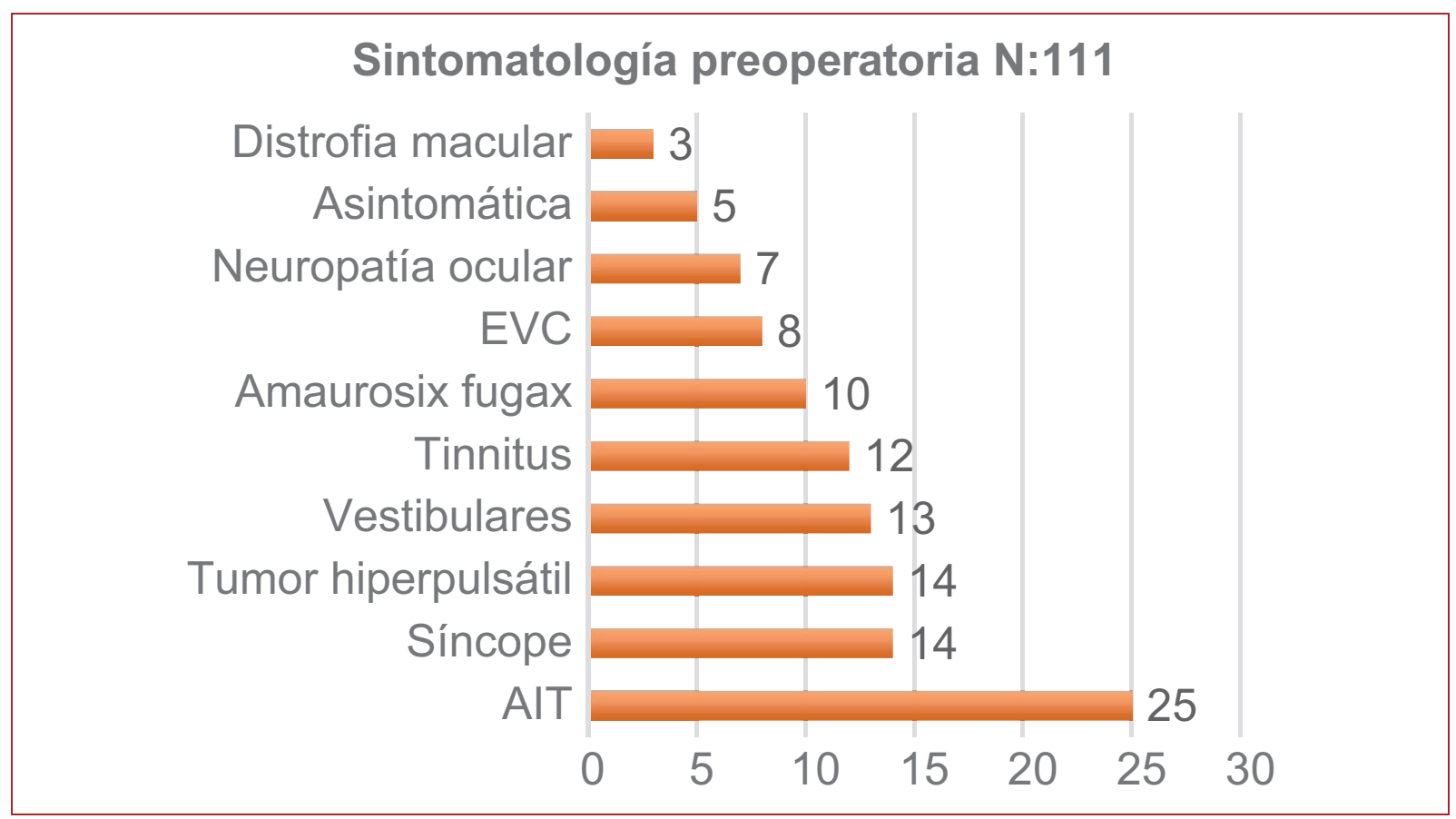

Figura 1. Sintomatología preoperatoria. (Fuente: Departamento de Angiología y Cirugía Vascular del Hospital de Especialidades del Centro Médico Nacional Siglo XXI del Instituto Mexicano del Seguro Social.)

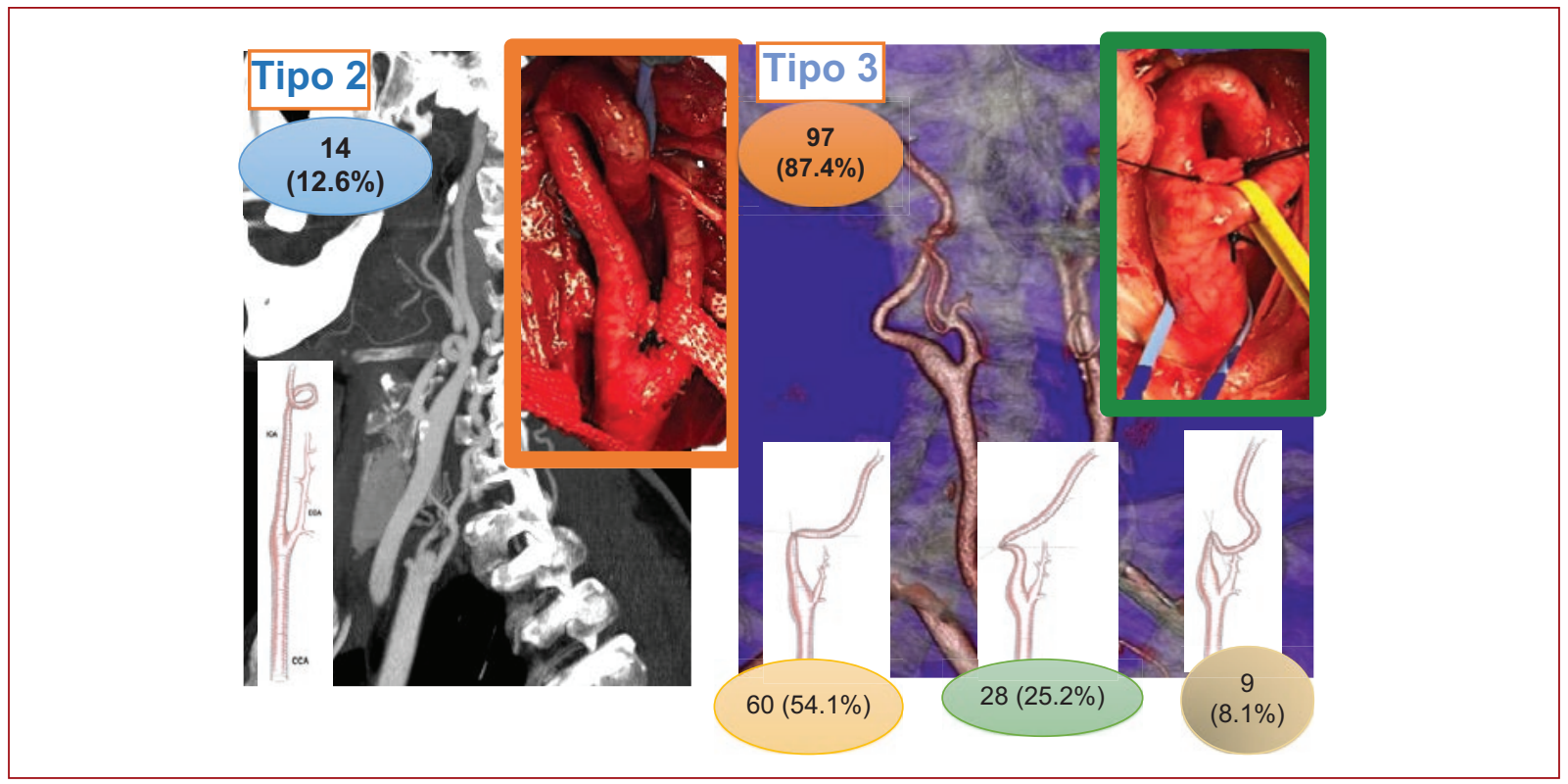

Figura 2. Tipos de dolicoarteriopatia de la arteria carótida interna. (Fuente: Departamento de Angiología y Cirugía Vascular del Hospital de Especialidades del Centro Médico Nacional Siglo XXI del Instituto Mexicano del Seguro Social.)

comorbilidades no cardiovasculares (patologías tiroideas, vasculares, enfermedades inflamatorias, neuropatías, cardiopatías no isquémicas) ( $p=0.005)$, de los
39 casos en los que hubo asociación a una de las comorbilidades previamente comentadas, el $90.7 \%$ no se curaron, en comparación con los 46 casos que no 


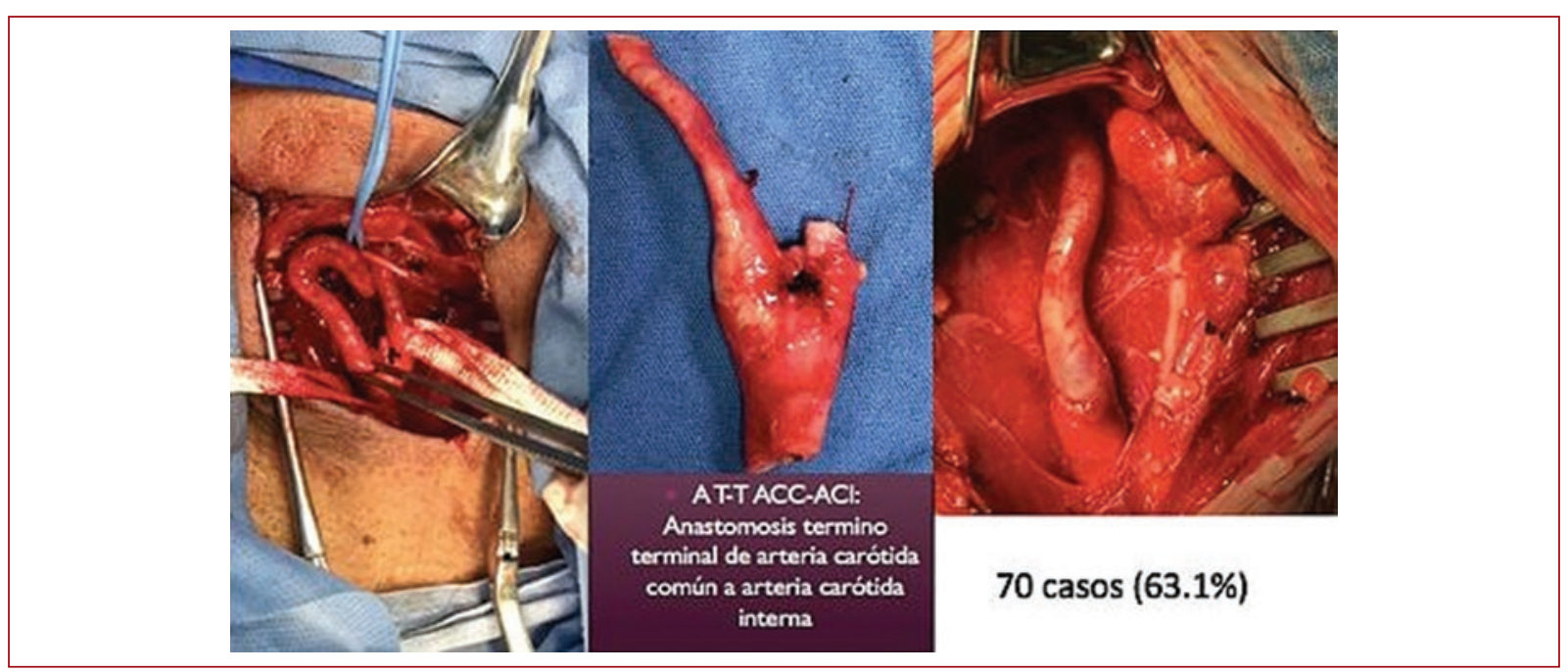

Figura 3. Resección de segmento redundante de la arteria carótida interna más anastomosis término-terminal de la arteria carótida común a la arteria carótida interna. (Fuente: Departamento de Angiología y Cirugía vascular del Hospital de Especialidades del Centro Médico Nacional Siglo XXI del Instituto Mexicano del Seguro Social.)

tenían dichas comorbilidades, que el $67.6 \%$ se curaron; la sintomatología preoperatoria $(p=0.082)$, se encontró que, de acuerdo al tipo de sintomatología, se puede predecir una mayor o menor corrección anatómica 0 hemodinámica sin presencia de complicaciones, y tenemos que cuando debutan con trastornos no hemisféricos tienen mayor posibilidad de corrección sin complicarse (curación mayor del 90\%), en especial cuando se trata del síncope, tumor hiperpulsátil y trastornos vestibulares; finalmente, el tiempo de pinzamiento $(p=0.045)$ fue otro factor predictor, un pinzamiento mayor de 30 minutos (RIC 23-35) se asoció a menor corrección hemodinámica/anatómica con riesgo de complicarse. Para la presencia de complicaciones, se encontraron tres variables con significancia estadística, IMC ( $p=0.003)$, endarterectomía simultánea $(p=0.037)$, sin embargo, a diferencia que para una corrección hemodinámica, el realizarla se asoció a que no se complicaran, y finalmente el pinzamiento, con una mediana de 20 minutos (RIC 17-28) $(p=0.005)$. El resto de las variables no fueron estadísticamente significativas (Tabla 1).

\section{Discusión}

Las DAC de la arteria carótida interna (DACl) pueden ocurrir hasta en un 10 a $25 \%$ de la población ${ }^{8}$. Esta incidencia es consistente con uno de los más grandes estudios, donde Pellegrino, et al., en 1998, examinaron 1,220 sujetos italianos y encontraron que 316 presentaban $\mathrm{DACl}$, correspondiente a una tasa de incidencia del $25.9 \%$. Metz, et al. (1961) y Weibel, et al. (1965) clasificaron las DACl en tres tipos: tipo 1, tortuosidad; tipo 2, asa o bucle, y tipo 3, acodamiento. Adicionalmente, Metz, et al. subdividieron el acodamiento en tres grados: I, leve (> 60 grados); II, moderado (30

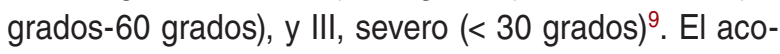
damiento o kinking es la anomalía morfológica más frecuente. Se presenta como una variante del tipo 2. En pacientes adultos con síntomas de insuficiencia cerebrovascular, a menudo se asocia con enfermedad ateromatosa a nivel de la bifurcación carotídea, con riesgo alto de estenosis u oclusión secundaria. En este estudio encontramos que de los 111 pacientes analizados, así como en las grandes series, la DAP más frecuente fue la de tipo 3 , presente en el $87.4 \%$ de los casos, y el grado I fue la encontrada en la mitad de los pacientes intervenidos. Hubo una asociación a enfermedad ateromatosa en el $42.3 \%$. La tortuosidad quizá fue subdiagnosticada al no ser incluida en este estudio. En cuestión de lateralidad, la afección bilateral estuvo presente en 61 casos (55\%) como ligera tendencia hacia el lado izquierdo, con un $25.2 \%$, comparado con el $19.8 \%$ del lado derecho. En las primeras series realizadas por Leipzing en 1986, reportaron más de 800 casos de tortuosidad o acodamientos de la arteria carótida interna como hallazgo en las arteriografías de rutina, sin predominio de género y bilaterales en la mayoría de los $\operatorname{casos}^{10}$. Nuestros resultados no están de acuerdo con los estudios que muestran una mayor prevalencia 


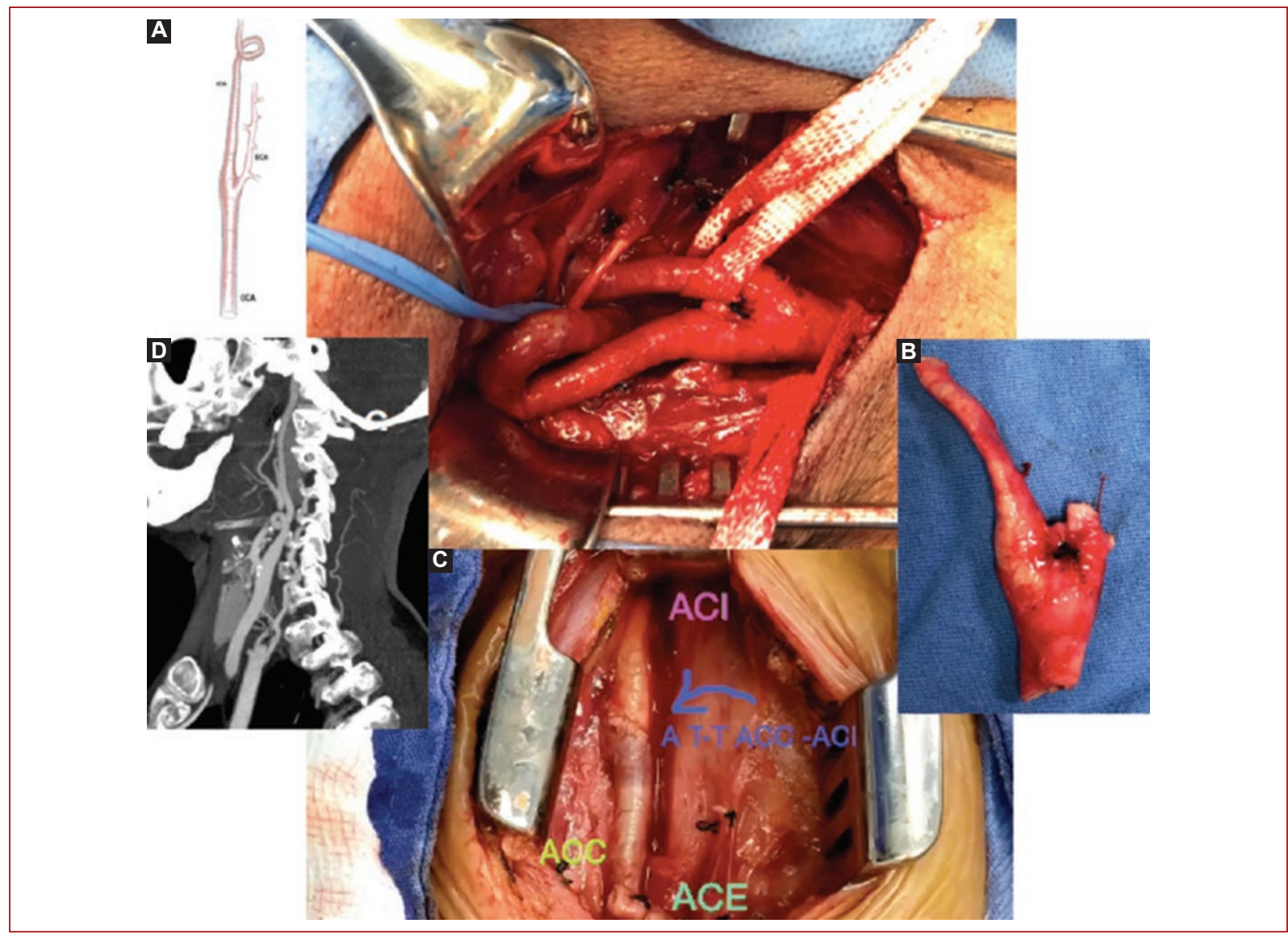

Figura 4. A: abordaje cervical izquierdo, donde se observa dolicoarteriopatía tipo 2. Referidas con cintas umbilicales la arteria carótida común y la arteria carótida externa, y con silastic (azul) la arteria carótida interna, la cual muestra un bucle cerca de la base del cuello. B: resección en bloque del segmento redundante, bulbo, ostium de la arteria carótida externa y segmento distal de la arteria carótida común. C: corrección anatómica con anastomosis término-terminal de la arteria carótida común a la arteria carótida interna con ligadura de la arteria carótida externa. D: angiotomografía de troncos supraaórticos, corte sagital donde se observa dolicoarteriopatía tipo 2. (Fuente: Departamento de Angiología y Cirugía vascular del Hospital de Especialidades del Centro Médico Nacional Siglo XXI del Instituto Mexicano del Seguro Social.)

de DAP en hombres (Weibel, et al., 1965; Koskas, et al., 1994) y en ancianos (Del Corso, et al., 1998; Pancera, et al., 1998; Pancera, et al., 2000). En este estudio, las mujeres de edad avanzada (> 60 años) se vieron afectadas con mayor frecuencia, el $78.4 \%$ de los casos comparado con el $21.6 \%$ para el sexo masculino, pero ambos sexos fueron igualmente afectados entre los pacientes menores de 60 años. Con respecto al resto de las variables demográficas analizadas, concuerdan con la investigación más recientes publicadas en el 2005 por Ballota, et al., quienes realizan el primer estudio prospectivo que habla acerca del tratamiento quirúrgico versus médico para el manejo de elongación de la arteria carótida interna variedad coiling o kinking en pacientes sintomáticos. Analizan un total de 182 casos, 91 sometidos a tratamiento quirúrgico y 90 a tratamiento médico ${ }^{11}$. De acuerdo a nuestro estudio, los tres principales factores de riesgo más prevalentes fueron la hipertensión arterial sistémica (78.4\%), el tabaquismo positivo (42.3\%) y la dislipidemia (39.6\%), lo que refuerza la teoría de que las $\mathrm{DACl}$ están teóricamente asociadas a factores de riesgo aterosclerótico, aunque, el análisis realizado por Jiulu Yu, et al. en 2017 refiere que algunos estudios han encontrado que estos factores de riesgo ateroesclerótico cardiovascular no ocurren con mayor frecuencia en pacientes con $\mathrm{DACl}$ que en pacientes sin $\mathrm{DACl}^{12}$; por lo tanto, el papel de la hipertensión u otros factores de riesgo cardiovascular en la génesis del DACI sigue siendo desconocida, además, el grosor miointimal y las placas carotídeas no muestran diferencias bilaterales significativas en comparación con los DACl monolaterales. Aunque el 
Tabla 1. Análisis bivariado de los predictores para la correción del segmento redundante de la arteria carótida interna $(\mathrm{n}=111)$

\begin{tabular}{|c|c|c|c|}
\hline \multicolumn{4}{|c|}{ Corrección hemodinámica } \\
\hline & No & Sí & $\mathbf{p}$ \\
\hline $\begin{array}{l}\text { Endarterectomía } \\
\text { No } \\
\text { Sí }\end{array}$ & $\begin{array}{l}2(2.6 \%) \\
5(14.7 \%)\end{array}$ & $\begin{array}{l}75(97.4 \%) \\
29(85.3 \%)\end{array}$ & 0.016 \\
\hline $\begin{array}{l}\text { Sangrado en ml, } \\
\text { mediana (RIC) }\end{array}$ & $\begin{array}{c}60 \\
(50-100 \%)\end{array}$ & $\begin{array}{c}33 \\
(20-50 \%)\end{array}$ & 0.027 \\
\hline \multicolumn{4}{|c|}{ Corrección anatómica } \\
\hline & No & Sí & $\mathbf{p}$ \\
\hline $\begin{array}{l}\text { VPS preoperatoria } \\
\mathrm{cm} / \mathrm{s} \text { mediana (RIC) }\end{array}$ & $\begin{array}{c}112 \\
(61-133 \%)\end{array}$ & $\begin{array}{c}130 \\
(102-155.75 \%)\end{array}$ & 0.049 \\
\hline \multicolumn{4}{|c|}{$\begin{array}{l}\text { Corrección anatómica y hemodinámica sin presencia de } \\
\text { complicaciones }\end{array}$} \\
\hline & No & Sí & $\mathbf{p}$ \\
\hline $\begin{array}{l}\text { IMC } \\
\text { Normal } \\
\text { Sobrepeso } \\
\text { Obesidad }\end{array}$ & $\begin{array}{l}13(31.7 \%) \\
21(65.6 \%) \\
36(94.7 \%)\end{array}$ & $\begin{array}{c}28(68.3 \%) \\
11(34.4 \%) \\
2(5.3 \%)\end{array}$ & 0.005 \\
\hline $\begin{array}{l}\text { Otras comorbilidades } \\
\text { no cardiovasculares } \\
\text { No } \\
\text { Sí }\end{array}$ & $\begin{array}{l}22(32.4 \%) \\
39(90.7 \%)\end{array}$ & $\begin{array}{c}46(67.6 \%) \\
4(9.3 \%)\end{array}$ & 0.005 \\
\hline $\begin{array}{l}\text { Síntomas al } \\
\text { diagnóstico } \\
\text { Asintomático } \\
\text { Síncope } \\
\text { EVC } \\
\text { Tumor hiperpusáltil } \\
\text { Amaurosis fugax } \\
\text { Distrofia macular } \\
\text { Neuropatía ocular } \\
\text { Tinnitus } \\
\text { Vestibulares } \\
\text { AIT }\end{array}$ & $\begin{array}{c}0(0 \%) \\
2(18.2 \%) \\
1(9.1 \%) \\
4(28.6 \%) \\
1(10 \%) \\
2(66.7 \%) \\
1(14.3 \%) \\
3(25) \\
1(7.7 \%) \\
11(44 \%)\end{array}$ & $\begin{array}{c}5(100 \%) \\
9(81.8 \%) \\
10(90.9 \%) \\
10(71.4 \%) \\
9(90 \%) \\
1(33.3 \%) \\
6(85.7 \%) \\
9(75 \%) \\
12(92.3 \%) \\
14(56 \%)\end{array}$ & 0.082 \\
\hline $\begin{array}{l}\text { Tiempo de } \\
\text { pinzamiento en min, } \\
\text { mediana (RIC) }\end{array}$ & $30(23-35 \%)$ & $\begin{array}{c}21.5 \\
(17.75-34.5 \%)\end{array}$ & 0.045 \\
\hline
\end{tabular}

\begin{tabular}{|l|c|c|c|}
\hline \multicolumn{4}{|c|}{ Presencia de complicaciones } \\
\hline & No & Sí & p \\
\hline IMC & & & 0.003 \\
\hline $\begin{array}{l}\text { Normal } \\
\text { Sobrepeso }\end{array}$ & $30(73.2 \%)$ & $11(26.8 \%)$ & \\
Obesidad & $24(75 \%)$ & $8(25 \%)$ & \\
\hline $\begin{array}{l}\text { Otras comorbilidades } \\
\text { no cardiovasculares } \\
\quad \text { No }\end{array}$ & $0(0 \%)$ & $38(100 \%)$ & 0.006 \\
$\quad$ Sí & $17(25 \%)$ & $51(75 \%)$ & \\
\hline $\begin{array}{l}\text { Tiempo de } \\
\text { pinzamiento, } \\
\text { mediana (RIC) }\end{array}$ & $20(17.7 \%)$ & $41(95.3 \%)$ & \\
\hline $\begin{array}{l}\text { Endarterectomía } \\
\text { simultánea } \\
\text { No } \\
\text { Sí }\end{array}$ & $30(23-35 \%)$ & 0.005 \\
\hline
\end{tabular}

AIT: ataque isquémico transitorio; HAS: hipertension arterial sistémica; IMC: índice de masa corporal; RIC: rango intercuartil; VPS: velocidad pico sistólica.

Comorbilidades no cardiovasculares: tiroideas, vasculares, inflamatorias,

cardiopatía no isquémica, neuomoapatías. (Fuente: Departamento de Angiología y Cirugía Vascular del Hospital de Especialidades del Centro Médico Nacional Siglo XXI del Instituto Mexicano del Seguro Social.) mecanismo tromboembólico secundario a las lesiones endoteliales debido a alteraciones del flujo local en el sitio de flexión arterial se ha considerado responsable de la aparición de muchos síntomas isquémicos en pacientes con elongación de la arteria carótida interna ${ }^{13,14}$, el mecanismo hemodinámico parece jugar un papel importante cuando el segmento redundante se estrecha tanto en condiciones neutras como dinámicas. Esta teoría está respaldada por estudios experimentales que demuestran que el flujo sanguíneo puede reducirse en un $40 \%$ con un ángulo de $60^{\circ}$ en el lado de la curva a lo largo del la arteria carótida interna y en un $60 \%$ con un ángulo de $30^{\circ 11,15}$, de modo que cuanto más pequeño sea el ángulo más hemodinámicamente significativo será el acodamiento y, en consecuencia, mayor será la reducción del flujo sanguíneo cerebral. Es por eso que en este estudio se intentó conocer qué factores influyen en una adecuada corrección anatómica, y se encontró que la velocidad sistólica máxima preoperatoria mostró una significancia estadística $(p=$ 0.049), de tal forma que, a mayor velocidad sistólica máxima con ensanchamiento espectral (RIC: 102$155.75 \mathrm{~cm} / \mathrm{s}$ ) hubo una mayor corrección anatómica posquirúrgica, comparado con los que tenían menor valor antes de ser operados VPS $112 \mathrm{~cm} / \mathrm{s}$ (RIC 61-133). No es fácil establecer una relación casual entre estas anomalías y el inicio clínico de los signos neurológicos, especialmente en los no hemisféricos, debido también a la coexistencia de enfermedad oclusiva aterosclerótica en el sitio de la bifurcación carotídea ${ }^{11}$. En nuestro estudio, como síntoma hemisférico, el ataque isquémico transitorio fue la sintomatología preoperatoria más frecuente $(22.5 \%)$, seguido de la amaurosis fugax $(9 \%)$ y el evento vascular cerebral (7.2\%). Como sintomatología no hemisférica, tanto el síncope como el tumor hiperpulsátil tuvieron una distribución homogénea, estando presentes cada uno en el $12.6 \%$ de casos, seguidos de los trastornos vestibulares, con el $11.7 \%$. En este estudio se evidenció que uno de los factores predictores para una adecuada corrección tanto anatómica como hemodinámica sin presencia de complicaciones fue justamente la sintomatología preoperaroria $(p=0.082)$, y se encontró que dependiendo del tipo de sintomatología se puede predecir una mayor corrección anatómica o hemodinámica sin presencia de complicaciones, teniendo que cuando debutan con trastornos no hemisféricos tienen mayor posibilidad de corrección sin complicarse (curación por encima del $90 \%$ ), en especial cuando se trata del síncope, tumor hiperpulsátil y trastornos vestibulares, no pudiendo extender está regla para la distrofia macular, en la que solamente el $33 \%$ 
de los casos se curaron. Para los hemisféricos no fue tan marcada la diferencia, y solo el $56 \%$ se curaron sin complicarse, comparado con solo el $44 \%$ que no se curaron. Otros factores predictores para una corrección tanto anatómica como hemodinámica sin presencia de complicaciones fueron el IMC ( $p=0.005)$, de los 111 pacientes, la obesidad estuvo presente en 36 , y solo se curó el $5.3 \%$, comparado con $31.7 \%$ de los pacientes con peso normal. El tener otras comorbilidades no cardiovasculares, tales como vasculares, enfermedades inflamatorias, neuropatías y cardiopatías no isquémica, también fue otro factor predictor $(p=0.005)$, ya que, de los 39 casos con otra comorbilidad, el $90.7 \%$ no se curó. Finalmente, el tiempo de pinzamiento $(p=0.045)$ fue otro factor predictor, y un pinzamiento mayor de 30 minutos se asoció a menor corrección hemodinámica/anatómica con riesgo de complicarse.

De acuerdo a las investigaciones más antiguas realizadas por Leipzig T, como lo describe en su artículo «The Tortuous or Kinked Carotid Artery: Pathogenesis and Clinical Considerations " ${ }^{10,16}$, esencialmente se utilizan tres técnicas en el manejo quirúrgico de segmento redundante de la arteria carótida interna (transposición, lisis de adherencias y resección o recolocación con anastomosis, sin embargo, se llegan a describir hasta siete técnicas para la reconstrucción de un segmento redundante):

1. La transposición arterial: En 1951, Riser, et al. describieron por primera vez la corrección de un acodamiento de la arteria carótida interna plicando el vaso a la parte inferior del músculo esternocleidomastoideo. Derrick no informó recurrencia de síntomas en pacientes observados 5-42 meses después de la transposición arterial ${ }^{10,2}$. Sin embargo, Vollmar, et al. criticaron estas técnicas al no eliminar el alargamiento arterial como factor patogénico que permite una torcedura recurrente ${ }^{17}$.

2. Lisis de adherencias: Las adherencias de estructuras cervicales a una arteria carótida interna redundante pueden producir o contribuir a la formación de un acodamiento. La lisis de cualquier banda de anclaje es crucial para el éxito quirúrgico, puede ser necesario incluso quitar la adventicia.

3. Procedimientos vasculares: Muchos avances se han desarrollado en la cirugía arterial carotídea desde que se introdujo en 1954 por Eastcott, et al. La resección segmentaria de la arteria carótida interna o arteria carótida común se realiza en un esfuerzo por abrir la angulación, o en realidad acortar la arteria elongada. Cuando el acodamiento está localizado o si hay implicación aterosclerótica en el codo del pliegue, la lesión puede ser resecada en bloque ${ }^{16-17}$ y la porción restante de la arteria carótida interna se anastomosa a la arteria carótida interna proximal u oblicuamente en la arteria carótida común. Debe tenerse en cuenta que una anastomosis de arteria carótida interna a carótida interna puede ser técnicamente difícil cuando los dos segmentos tienen un tamaño diferente ${ }^{18-20}$. De acuerdo a esta investigación, en el Servicio de Angiología y Cirugía Vascular del Centro Mexicano Nacional Siglo XXI, se opta por la combinación de las técnicas previamente referidas, eligiendo el tipo de técnica de reconstrucción de acuerdo a la planeación preoperatoria con la angiotomografía de troncos supraaórtiocos, los hallazgos transquirúrgicos, así como la experiencia y las habilidades individuales del cirujano vascular. Así, tenemos que la anastomosis término-terminal de la arteria carótida común a la arteria carótida interna con o sin ligadura de arteria carótida externa, con 70 casos (63.1\%), seguida de la anastomosis término-terminal de la arteria carótida común a la arteria carótida común, con 26 casos (23.4\%) fueron las dos técnicas más empleadas. El reimplante de la arteria carótida interna, la anastomosis término-terminal de la arteria carótida interna, con 3 casos (2.7\%) para cada técnica, y la interposición de injerto protésico, con 2 casos (1.8\%), se realizaron en una minoría de pacientes. No se optó por estos tipos de reconstrucción al ser técnicamente más difícil de realizar la anastomosis, asociarse a mayor tiempo de pinzamiento (45 minutos) y sangrado transquirúrgico $(300 \mathrm{ml})$. Coincidió que estos tipos de técnicas fueron empleadas solamente en paciente con bifurcación baja (C5-C6). Las técnicas de plicatura a estructuras vecinas o plicatura posterior transversa de la arteria carótida interna no se realizan en este centro ${ }^{21-23}$.

La necesidad ocasional de corregir el segmento redundante de la arteria carótida interna después de la endarterectomía carotídea todavía es controvertida ${ }^{24}$. Aunque se han sugerido varias opciones técnicas, el impacto en el resultado perioperatorio sigue siendo incierto, y falta un seguimiento clínico a largo plazo. El acortamiento por resección tiene un riesgo teórico de anastomosis torcida y posterior trombosis de la arteria carótida interna, de acuerdo a lo que la literatura internacional refiere ${ }^{4,21-27}$. En el presente estudio, justamente, la endarterectomía carotídea simultánea fue un factor predictor con significancia estadística $(p=0.016)$ para la corrección hemodinámica favorable, teniendo que, de los 77 casos en los que no se realizó endarterectomía simultánea, solo el $2.6 \%$ no tuvo una corrección hemodinámica, comparado con el $14.7 \%$ en los que sí se realizó dicho procedimiento de forma 
simultáneamente. Sin embargo, cuando se analizó como factor predictor para presentar complicación posquirúrgica, continuó mostrando significancia estadística $(p=0.037)$, pero de los 77 casos en los que no se realizó, el $22.1 \%$ se complicaron, comparado con el $5.9 \%$ en los que sí se realizó. En nuestro centro, cuando el porcentaje de estenosis carotídea es mayor al 60 a $70 \%$ asociado a predominio cálcico en la morfología de la placa y partiendo de las investigaciones realizadas por Vannix, et al. ${ }^{7,28-35}$, que consideran que la endarterectomía debilita aún más la pared en el sitio de acodamiento, con la finalidad de acortar tiempos de pinzamiento y de procedimiento, en general, no se realiza endarterectomía simultánea y se reseca en bloque la lesión junto con el segmento redundante de la arteria carótida interna para posterior anastomosis término-terminal de la arteria carótida común a la interna con ligadura de ACE, de tal forma que se corrige el defecto anatómico y a su vez se elimina al $100 \%$ la placa calificada que condiciona estenosis carotídea. Dichos resultados se ven reflejados al mes de seguimiento con el rastreo ultrasonográfico Doppler dúplex, donde se evidencia una adecuada corrección hemodinámica con normalización de la velocidad sistólica máxima, como se pudo observar en el análisis estadístico, donde, de los 111 procedimientos realizados, 104 (93.7\%) tuvieron una corrección hemodinámica.

\section{Conclusión}

La corrección del segmento redundante de la $\mathrm{ACl}$ es una técnica segura y eficaz. El realizar la endarterectomía simultánea es un factor predictivo tanto para una corrección hemodinámica como para la presencia de complicaciones. De tal forma que no realizarla de forma simultánea predice una mayor corrección hemodinámica, por lo que se sugiere la resección en bloque de la lesión con placa ateroesclerótica junto con el segmento redundante. Sin embargo, predijo una menor tasa de que se compliquen, sin considerar la corrección anatómica y hemodinámica. Habrá que extender el estudio para que permita establecer los criterios para la realización de endarterectomía simultánea o resección en bloque de acuerdo a morfología de la placa ateroesclerótica.

La VPS preoperatoria es un factor predictor para corrección anatómica, de tal forma que a mayor repercusión hemodinámica preoperatoria mayor será el éxito en cuestión de corrección estructural.

Para una corrección tanto anatómica como hemodinámica sin presencia de complicaciones se encontraron cuatro variables predictoras estadísticamente significativas:
1. IMC: se encontró que la obesidad se asoció a que no se corrigiera y se complicaran, comparado con los que tienen un peso normal.

2. La sintomatología preoperatoria: se puede predecir una mayor corrección anatómica o hemodinámica sin presencia de complicaciones, teniendo que cuando debutan con trastornos no hemisféricos tienen mayor posibilidad de corrección sin complicarse, en especial cuando se trata del síncope, tumor hiperpulsátil y trastornos vestibulares, en comparación a los trastornos hemisféricos.

3. Asociación a otras comorbilidades no cardiovasculares, tales como patologías tiroideas, vasculares, enfermedades inflamatorias, neuropatías, cardiopatías no isquémicas: se asoció a que no se corrigiera anatómica o hemodinámicamente y se complicaran.

4. Tiempo de pinzamiento: un pinzamiento a partir de los 30 minutos se asoció a que no se corrigiera anatómica o hemodinámicamente y se complicaran.

Para la presencia de complicaciones, se identificaron 3 predictores: 1) IMC; 2) realizar endarterectomía simultánea, y 3) pinzamiento carotídeo, a partir de los 20 minutos se asoció a presencia de complicaciones.

\section{Conflicto de intereses}

Los autores declaran que no hay conflicto de intereses económicos, laborales, étnicos ni personales, relacionados con este artículo.

\section{Responsabilidades éticas}

Protección de personas y animales. Los autores declaran que para esta investigación no se han realizado experimentos en seres humanos ni en animales.

Confidencialidad de los datos. Los autores declaran que han seguido los protocolos de su centro de trabajo sobre la publicación de datos de pacientes.

Derecho a la privacidad y consentimiento informado. Los autores han obtenido el consentimiento informado de los pacientes y/o sujetos referidos en el artículo. Este documento obra en poder del autor de correspondencia.

\section{Bibliografía}

1. Beigelman R, Izaguirre A, Robles M, Grana D, Ambrosio G, Milei J. Are Kinking and Coiling of Carotid Artery Congenital or Acquired? Angiology. 2010;61(1):107-12.

2. Michalinos A, Chatzimarkos M, Arkadopoulos N, Safioleas M, Troupis T. Anatomical Considerations on Surgical Anatomy of the Carotid Bifurcation. Hindawi Publishing Corporation Anatomy Research International. 2016;2016:6907472. 
3. Del Corso L, Moruzzo D, Conte B, Agelli M, Romanelli AM, Pastine F, et al. Tortuosity, Kinking, and Coiling of the Carotid Artery: Expression of Atherosclerosis or Aging? Angiology. 1998:49(5):361-71.

4. Poorthuis M, Brand E, Toorop R, Moll F, de Borst G. Posterior transverse plication of the internal carotid artery to correct for kinking. Journal of vascular surgery. J Vasc Surg. 2014;59(4):968-77

5. Illuminati G, Ricco J, Caliò F, D'Urso A, Ceccanei G, Vietri F. Results in a consecutive series of 83 surgical corrections of symptomatic stenotic kinking of the internal carotid artery. Surgery. 2008;143(1):134-9.

6. Pancera P, Ribul M, Presciuttini B, Lechi A. Prevalence of carotid artery kinking in 590 consecutive subjects evaluated by Echocolordoppler. Is there a correlation with arterial hypertension? J Inter Med. 2000; 248(1):7-12.

7. Vannix RS, Joergenson EJ, Carter R. Kinking of the Internal Carotid Artery. Clinical Significance and Surgical Management. Am J Surg 1977:134(1):82-9

8. Cvetko E. "Concurrence of bilateral kinking of the extracranial part of the internal carotid artery with coiling and tortuosity of the external carotid artery--a case report. Rom J Morphol Embryol. 2014;55:433-5.

9. Zenteno M, Viñuela F, Moscote-Salazar LM, Miranda A, Zavaleta R, Flores Z, et al. Clinical implications of internal carotid artery tortuosity, kinking and coiling: a systematic review. Romanian Neurosurgery. 2014;21(1):51-60

10. Leipzig T, Dohrmann G. The Tortuous or Kinked Carotid Artery: Pathogenesis and Clinical Considerations. A Historical Review. Surg Neurol. 1986;25:478-86.

11. Ballotta E, Thiene G, Baracchini C, Ermani M, Militello C, Da Giau G, Barbon B, Angelini A. Surgical vs medical treatment for isolated internal carotid artery elongation with coiling or kinking in symptomatic patients: a prospective randomized clinical study. J Vasc Surg. 2005 42:838-46.

12. Yin L, Li Q, Zhang L, Qian W, Liu X. Correlation between cervical artery kinking and white matter lesions. Clin Neurol Neurosurg. 2017;157:51-4.

13. Mukherjee $D$, Inahara $T$. Management of the tortuous internal carotid artery. Am J Surg. 1985;149:651-5

14. Koskas F, Bahnini A, Walden R, Kieffer E. Stenotic coiling and kinking of the internal carotid artery. Ann Vasc Surg. 1993;7:30-40.

15. Derrick JR, Estess M, Williams D. Circulatory dynamics in kinking of carotid artery. Surgery. 1965;58:381-3.

16. Hurwitt ES, Carton CA, Fell SC, Kessler LA, Seidenberg B, Shapiro JH Clinical evaluation and surgical correction of obstruction in the branches of the aortic arch. Ann Surg. 1960;152:472-84.

17. Castilla JM, Delgado $P$, Martín V, Fernández-Arconada O, Martín-Santidrián MA, Rodríguez-Salazar A. Morbimortality in carotid endarterectomy Neurocirugia (Astur). 2005;16(2):93-107.

18. Fatic N, Jaffer U, Ivana S, Gordana GM, Markovic D, Kostic D, et al. Bilateral Internal Carotid Artery Occlusion, External Carotid Artery Stenosis, and Vertebral Artery Kinking: May It Be Asymptomatic? Ann Vasc Surg. 2017;44:416.e5-416.e8.
19. Martins HF, Mayer A, Batista P, Soares F, Almeida V, Pedro AJ, et al. Morphological changes of the internal carotid artery: prevalence and characteristics. A clinical and ultrasonographic study in a series of 19804 patients over 25 years old. Eur J Neurol. 25(1):171-7.

20. Togay-lşikay C, Kim J, Betterman K, Andrews C, Meads D. Tesh P, et al. Carotid artery tortuosity, kinking, coiling: stroke risk factor, marker, or curiosity? Acta Neurol Belg. 2005;105(2):68-72.

21. Garcia-Alva R, Lopez-Pena G, Ricárdez GR, De los Ríos J, Ruben-Castillo C, Pérez-Corrales ES, Elenes-Sánchez E, et al. Utilidad de la volumetría de los tumores del cuerpo carotídeo como predictor de sangrado transoperatorio y tiempo quirúrgico. Rev Mex Angiol. 2019:47(3):1-5

22. Zenteno M, Viñuela F, Moscote-Salazar LR, Alvis-Miranda HR. Clinical implications of internal carotid artery tortuosity, kinking and coiling: a systematic review. Romanian Neurosurgery. 2014;21(1):51-60.

23. Noh SM, Kang HG. Clinical significance of the internal carotid artery angle in ischemic stroke. Sci Rep. 2014;9(1):4618.

24. Saba L, Mallarini G. Correlation between kinking and coiling of the carotid arteries as assessed using MDCTA with symptoms and degree of stenosis. Clin Radiol. 2010;65:729-34

25. Yu J, Qu L, Xu B, Wang S, Li C, Xu Y, et al. Current Understanding of Dolichoarteriopathies of the Internal Carotid Artery: A Review. Current Understanding of Dolichoarteriopathies of the Internal Carotid Artery: A Review. International Journal of Medical Sciences. [Internet]. 2010;14(8):772-84

26. Paulsen F, Tillmann B, Christofides C, Richter W, Koebke J. Curving and looping of the internal carotid artery in relation to the pharynx: frequency, embryology and clinical implications. J Anat. 2000;197(3):373-81.

27. Yu C, Xiong J, Dai C, Guan B, Li Y. Independent risk factors for morphological abnormalities of the internal carotid arter. Acta Cardiol. 2013:68(5):481-7.

28. van Alphen HA. Kinking of the carotid artery: indications for surgery and surgical procedure. Clin Neurol Neurosurg. 1978;80(2):92-9

29. Kaplan ML, Bontsevich DN, Shilko SV. Role of local hemodynamic abnormalities at pathologic kinking of the carotid arteries in development of vascular cerebral insufficiency. Russ J Biomech. 2015;19(1):5-19.

30. Yu K, Zhong T, Li L, Wang J, Chen Y, Zhou H. Significant Association between Carotid Artery Kinking and Leukoaraiosis in Middle-Aged and Elderly Chinese Patients. J Stroke Cerebrovasc Dis. 2015; 24(5):1025-31.

31. Bookland M, Loftus C. Surgical Management of Extracranial CarotidArtery Disease. Surgical Management of Extracranial Carotid Artery Disease. Sección 3. Surgery for Cerebral Stroke. 2012;27(1):793-805.

32. Sethi S, Lau J, Godbold J, Gustavson S, Olin J. The S. Curve: A novel morphological finding in the internal carotid artery in patients with fibromuscular displasia. Vasc Med. 2014;19(5) 356-62.

33. Harrison J, Davalos PA. Cerebral ischemia Suurgical procedure in cases due to tortuosity and bulking of the cervical vessels. Arch Surg. 1962;84:109-12.

34. Anjali K, Pedram A. Estrogen and Progesterone Inhibit Vascular Smooth Muscle Proliferation. Endocrinology. 1997;138(8):3330-39.

35. Pellegrino L, Prencipe G. Dolicho-arteriopathies (kinking, coiling, tortuoosity) of the carotid arteries: study by color Doppler ultrasonography. Minerva Cardioangiologist. 1998;46:69-76. 